\title{
The electrical properties of muscle fibre membranes in dystrophia myotonica and myotonia congenita
}

\author{
A. J. MCCOMAS AND K. MROŻEK \\ From the Department of Physiology, University of Newcastle upon Tyne, and \\ Muscular Dystrophy Laboratories, Newcastle General Hospital
}

The disorder of skeletal muscle known as myotonia has repeatedly attracted the interest of clinical neurophysiologists. The condition is characterized by a prolonged contraction of muscle fibres following voluntary exertion; alternatively it may be induced by direct or indirect electrical stimulation of muscle or by mechanical percussion of the muscle belly. The condition may be an accompanying manifestation of several muscle disorders. In dystrophia myotonica the muscle fibres are dystrophic with evidence of necrosis and degeneration; in myotonia congenita the fibres show little sign of pathology though they are commonly hypertrophied; in paramyotonia the condition is induced by cold. Finally myotonia has been reported as a feature of adynamia episodica (Van der Meulen, Gilbert, and Kane, 1961) and polyneuritis (Worster-Drought and Sargent, 1952). The condition also occurs in an inherited form in goats (Kolb, 1938; Brown and Harvey, 1939) and is a frequent finding in hereditary mouse dystrophy (McIntyre, Bennett, and Brodkey, 1959; Lenman, 1963; McComas and Mossawy, 1965).

It was the achievement of Lindsley and Curnen (1936) to resolve previous arguments by showing unequivocally that the prolonged myotonic contractions were accompanied by electrical activity in the affected muscles and they attributed this to continuing motor nerve activity. Evidence of a central nervous system component in myotonia was also found by Denny-Brown and Nevin (1941) and they showed that this was responsible for the phenomenon of afterspasm. On the other hand, the most striking electrophysiological feature of myotonic muscle, the rapid burst of action potentials which gradually lessens in frequency (dive bomber potentials), is a property of the muscle fibre membrane itself, since studies in man and in animals have shown that it resists not only motor nerve block but also curarization (Brown

'Present address: Department of Neurology, Medical School, Warsaw. and Harvey, 1939; Floyd, Kent, and Page, 1955; McComas and Mossawy, 1965).

The technique of recording the electrical activity of single muscle fibres with micro-electrodes is one which is clearly suited to a study of the myotonic muscle fibre membrane. To date three such studies have been reported on patients with dystrophia myotonica or myotonia congenita. Observations have been made on the resting membrane potential and the presence or absence of 'background' electrical activity; unfortunately, however, the results have differed in the three studies. Thus a fall of resting potential in myotonic fibres was found by Hofmann, Alston, and Rowe (1966) but not by Riecker, Dobbelstein, Röhl, and Bolte (1964) or by Norris (1962; see, however, Discussion).

On the other hand repetitive depolarization potentials in myotonic fibres were noted by Norris (1962) but not by Riecker et al. (1964). In their initial study Hofmann and Rowe (1966) were also unable to detect background activity though it is described in their subsequent report (Hofmann et al., 1966). In the present study we have investigated two patients with myotonia congenita and two with dystrophia myotonica. We believe that the discrepancies in previous reports with regard to resting potential are due to the fact that it has different values in the two conditions. We have also studied the repetitive electrical activity which was found to be a much more prominent feature of myotonia congenita than dystrophia myotonica. Finally, we include the results of experiments in which the excitability of the muscle fibre membrane was determined by direct stimulation through an intracellular micro-electrode.

\section{METHODS}

PATIENTS Four male patients were investigated; they were volunteers to whom the nature of the study had been explained beforehand. They had been diagnosed as suffering from either myotonia congenita or dystrophia 
myotonica by a consultant neurologist (Dr. J. N. Walton or Dr. J. B. Foster). All the patients presented the classical features of their conditions and in no case was the diagnosis in doubt.

J.L. was a blacksmith's striker, aged 54, with dystrophia myotonica. He had noticed difficulty in relaxing his grip during the previous two years and this was worse in cold weather. He had a typical dystrophic facies with frontal baldness but there was no evidence of testicular atrophy or cataract. There was marked weakness of the sternomastoids and serratus anterior muscles and of the long flexors of the fingers; the biceps, triceps, and quadriceps were slightly weak. Myotonia could be demonstrated in hand muscles following willed effort and percussion.

$\boldsymbol{K} . \boldsymbol{R}$. was a roadman, aged 28 , with dystrophia myotonica. He had complained of weakness and inability to relax his grip during the previous three years. He had frontal baldness and lenticular opacities in association with a dystrophic facies. There was obvious weakness together with some wasting of the sterno-mastoids and the more distal muscles of the limbs; the left quadriceps was slightly weak in addition. Myotonia on gripping was marked but percussion myotonia could not be elicited.

G.H. was a paint sprayer and T.L. was a plumber; they were aged 41 and 20 respectively and both had myotonia congenita. The myotonia was severe and was associated with moderate muscular hypertrophy; there was no other evidence of somatic disease.

Electromyographic examination of all four patients revealed profuse myotonic discharges; in the patients with dystrophia myotonica there were, in addition, large numbers of low-voltage, short duration, polyphasic potentials.

MICRO-ELECTRODE TECHNIQUE In each patient the microelectrode investigation was made on the left rectus femoris muscle and a muscle biopsy was subsequently taken for histological examination. The preparation of the muscles and the stimulating and recording systems have been described elsewhere (McComas, Mrożek, Gardner-Medwin, and Stanton, 1968).

STATISTICAL TREATMENT OF RESUlTS Throughout the text, mean values have been presented with standard errors of the mean. The significance of differences between means has been calculated using the $t$ test.

\section{RESULTS}

DYSTROPHIA MYOTONICA Myotonic activity Only three fibres with myotonic discharges were encountered in the two patients and none of these fibres could be satisfactorily impaled with a microelectrode.

Resting membrane potentials Successful penetrations were made in $\mathbf{4 0}$ fibres of the first patient (K.R.) and in 54 fibres of the second (J.L.); the mean resting potentials were $68.3 \pm 1.6 \mathrm{mV}$ and $73.6 \pm 1.5 \mathrm{mV} \bar{z}$ respectively. The frequencies of the observed resting $\stackrel{\mathbb{D}}{\subseteq}$ potential values in the combined populations of $\bar{D}$ fibres are shown in Figure 1A. The results are noteworthy in two respects. Firstly, both mean values are
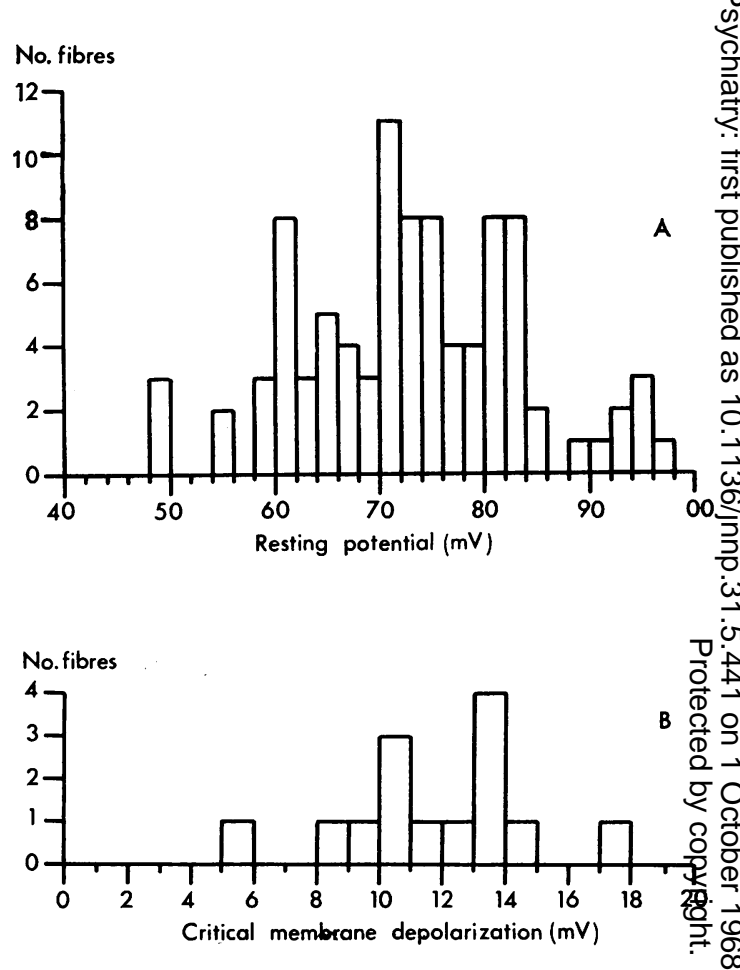

FIG. 1. A. Resting membrane potentials of 94 fibres in two patients with dystrophia myotonica; mean, $71.4 \pm 1 \cdot 1$ $m V$. B. Critical membrane depolarization in dystrophia myotonica; mean, $11 \cdot 3 \pm 0 \cdot 8 \mathrm{mV}$ (14 fibres).

significantly lower than those found in control subjects by McComas et al. (1968): thus in 134 con- 윽 trol fibres the mean resting potential was $83.6 \pm 0.50 \frac{3}{J}$ $\mathrm{mV}(P=<0.001)$. Secondly, the large scatter of the observations (48 to $95 \mathrm{mV}$ ) deserves comment. If, in a patient with dystrophia myotonica, a fall in the $\overline{3}$ resting potential of a fibre is indicative of dystrophic ? involvement (see Discussion) then those fibres with $\frac{0}{3}$ low potentials are likely to be the most severely affected, while others, with higher resting potentials, 을 probably retain some degree of normal morphology.

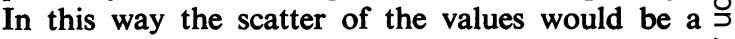
reflection of the pathological heterogeneity existing $\frac{D}{2}$ among fibres in a dystrophic muscle at a given time.

Intracellular stimulation In a total of 21 fibres in $\mathrm{N}$ the two subjects it was possible to study the effects of intracellular stimulation. It was found that the 


\section{TABLE I}

MEMBRANE CONSTANTS OF MUSCLE FIBRES IN DYSTROPHIA MYOTONICA AND MYOTONIA CONGENITA, TOGETHER WITH CORRESPONDING VALUES IN CONTROL SUBJECTS (MCCOMAS et al., 1968)

\begin{tabular}{lllll} 
& $\begin{array}{l}R . P . \\
(m V)\end{array}$ & $\begin{array}{l}T_{\mathrm{m}} \\
(\mathrm{msec})\end{array}$ & $\begin{array}{l}R_{\text {in }} \\
\left(\times 10^{5} \Omega\right)\end{array}$ & $\begin{array}{l}C . M . D . \\
(m V)\end{array}$ \\
\hline Control & $83.6 \pm 0.5$ & $6.3 \pm 0.4$ & $2.7 \pm 0.2$ & $11.8 \pm 0.4$ \\
& $(134)$ & $(19)$ & $(19)$ & $(37)$ \\
Dystrophia & $71.4 \pm 1.1 *$ & $5.0 \pm 0.3 * 2.3 \pm 0.2$ & $11.3 \pm 0.8$ \\
myotonica & $(94)$ & $(20)$ & $(20)$ & $(14)$ \\
Myotonia congenita & $82.0 \pm 0.8$ & - & - & $8.8 \pm 0.8 *$
\end{tabular}

Each result has been expressed as mean \pm standard error of mean, with the number of observations given in brackets. Asterisks indicate values significantly different $(P=<0.01)$ from controls. R.P., resting potential; $\mathbf{T}_{\mathrm{m}}$, membrane time constant $; \mathbf{R}_{\mathrm{in}}$, input resistance : C.M.D., critical membrane depolarization.

critical membrane potential, like the resting membrane potential, had also been reduced in many fibres; as a consequence the mean critical membrane depolarization $(11.3 \pm 0.8 \mathrm{mV}$; Fig. 1B) was not significantly different from the control mean (11.8 \pm $0.5 \mathrm{mV}$; McComas et al., 1968). The membrane time-constants and the input resistances were also measured (Table I) and in general were slightly shorter than the control values obtained byMcComas et al. (1968).

MYOTONIA CONGENITA General features In contrast to the observations in dystrophia myotonica (see above) the muscles of the two patients with myotonia congenita displayed abundant myotonic activity. Thus approximately two-thirds of the fibres investigated showed high frequency discharges of action potentials following impalement with microelectrodes. Unfortunately, the twitching associated with the myotonia made investigation of individual fibres difficult and it was usually not possible to perform intracellular stimulation satisfactorily. Instead we have limited our observations to the resting membrane potential and to the membrane changes associated with the myotonic discharges.

Myotonic activity The frequency of the myotonic potentials was greatest immediately following microelectrode impalement and subsequently declined; the initial firing rates varied between $20 / \mathrm{sec}$ and $100 / \mathrm{sec}$. Similar discharge frequencies were noted by Norris (1962) in an in vivo study of limb muscle and by Hofmann et al. (1966) in their in vitro investigation of intercostal fibres. In contrast to these observations, Riecker et al. (1964) were unable to detect myotonic discharges in patients with myotonia congenita and attributed this to the high mechanical stability of their recording system.

Our own observations seem pertinent to this matter. On the one hand it was obvious from inspection of the incessantly twitching surface of a muscle that many fibres possessed myotonic activity which was quite independent of external influences. On the other hand all the recorded activity exhibited pre-potentials before the spikes of the myotonic action potentials (Fig. 2) and this clearly showed that the activity commenced at the micro-electrode tip. Since the micro-electrodes were inserted randomly into the muscle, the myotonic activity cannot have arisen at some constant morphological feature of the fibres (for example, the motor end-plates); instead, the only reasonable interpretation is that the microelectrode itself was initiating the myotonic dis-

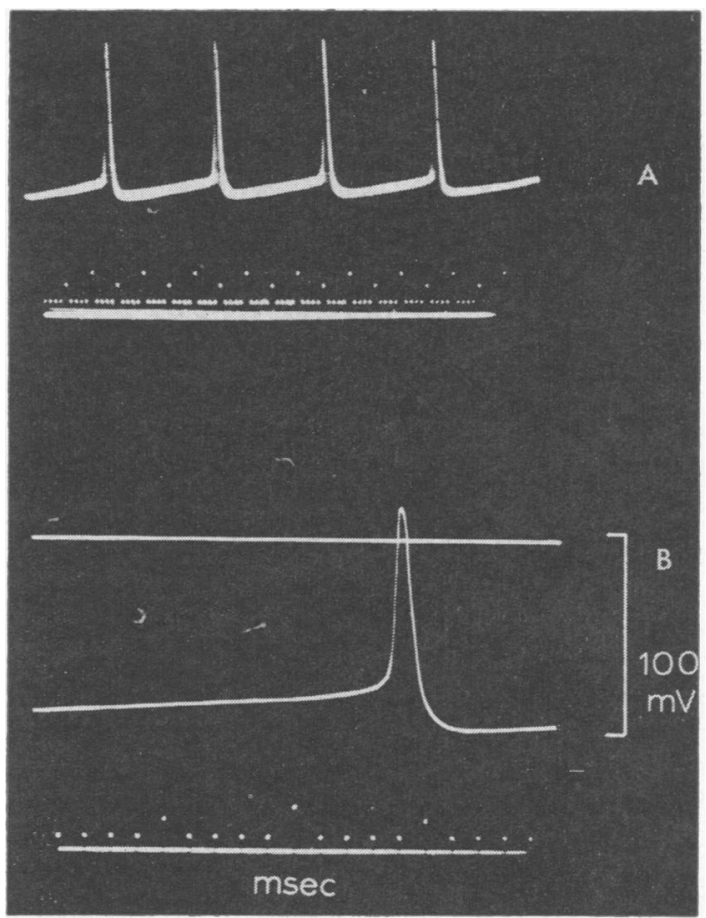

FIG. 2. Insertion activity in two fibres of a patient with myotonia congenita. The muscle fibre corresponding to the upper trace $(A)$ had a resting potential of $74 \mathrm{mV}$, a critical membrane depolarization of $5 \mathrm{mV}$ and an action potential of $82 \mathrm{mV}$. For the lower trace $(B)$ the values were 92, 13, and $110 \mathrm{mV}$ respectively.

charges and that the latter were therefore examples of 'insertion activity'.

By measuring the sizes of the pre-potentials in the discharging fibres it has been possible to obtain values for the critical membrane depolarizations (Fig. 3B). The results were found to range from $3 \mathrm{mV}$ to $13 \mathrm{mV}$; however, the low values were obtained at 

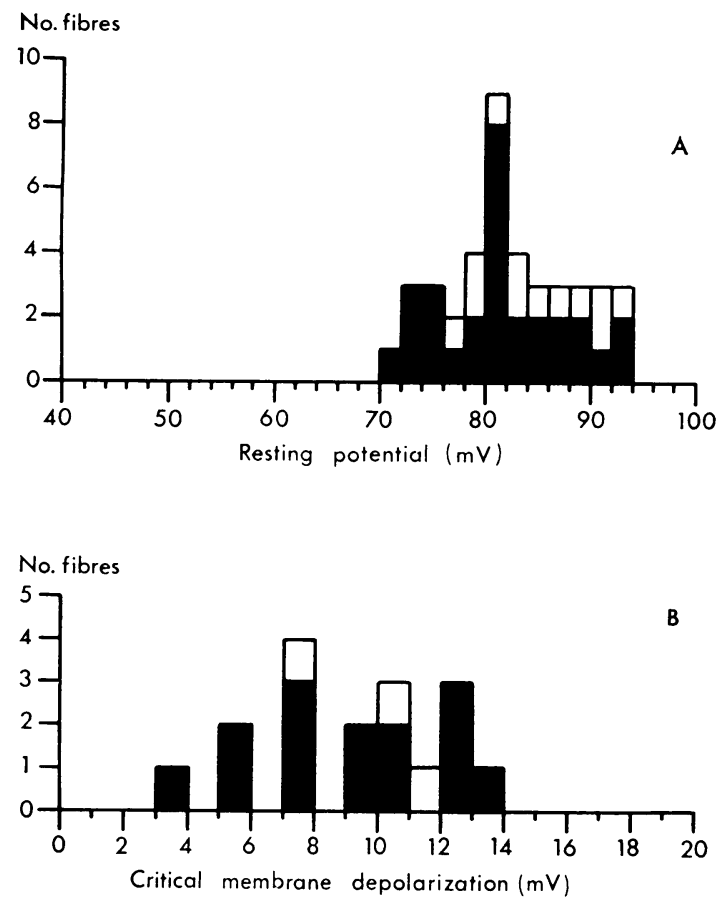

FIG. 3. A. Resting membrane potentials of 42 fibres in two patients with myotonia congenita; mean, $82.0 \pm 0.9$ $m V$. Filled columns indicate values in fibres with insertion activity (see text). B. Critical membrane depolarizations in myotonia congenita; mean, $8.8 \pm 0.8 \mathrm{mV}$ (17 fibres). Filled columns indicate values obtained from pre-potentials during insertion activity (see text); open columns correspond to results of intracellular stimulation.

times when the fibres were undergoing progressive depolarization and must therefore be interpreted with caution (for example, Fig. 2, upper; see Discussion).

Resting membrane potentials The myotonic discharges posed several problems for the successful determination of resting membrane potential. Firstly, the twitching associated with the activity caused the electrodes to become easily dislodged from the fibres. Alternatively, electrode tips might remain within the fibres but the resting membrane potentials would fall progressively; the likeliest explanation was that the twitching caused the membranes to tear around the electrodes. The third difficulty was in deciding the correct reference potential to use for measuring the resting membrane potential, since in the active fibres the membranes were continually alternating between pre-potentials and spikes. To resolve this difficulty we have followed Norris (1962) by measuring the membrane potential immediately after the spike; in our experience this value corresponds to the mem- brane potential encountered on first penetrating the fibre.

The number of resting potential measurements made in the two subjects has inevitably been small due to the difficulties described above. In the first subject (T.L.) the mean resting potential was 82.5 $1.8 \mathrm{mV}$ (20 fibres), while in the second patient (G.H.) a mean of $81.1 \pm 0.8 \mathrm{mV}$ was obtained (22 fibres). The pooled observations have been presented in Fig. 3A where a distinction has been made between the resting potentials of active and inactive fibres. In view of the difficulties experienced in determining resting potentials, separate means have also been computed for the active and inactive fibres $(81 \cdot 1$ 1.2 and $84.0 \pm 1.3 \mathrm{mV}$ respectively). However, it was found that in both instances the mean resting potentials were not significantly different from the control mean of $83.6 \pm 0.50 \mathrm{mV}$ found by McComas et al. (1968).

\section{DISCUSSION}

RESTING POTENTIAL MEASUREMENTS In dystrophia myotonica the resting potentials were found to be significantly reduced compared with the values obo tained in this laboratory for fibres from contro muscles (McComas et al. 1968). In this respect the results are similar to those of Hofmann et al. (1966) who, studying intercostal muscle in vitro, found reduced mean membrane potential in their 10 patients with myotonia, of whom nine were diagnosed a having dystrophia myotonica. A significant reduce tion in resting potential has also been noted in excised human intercostal muscle in cases of Duchenne dystrophy (Ludin, 1967) and also in several in vivo studies of hereditary mouse dystrophy (Kleeman, Partridge, and Glaser, 1961; Lenman, 1963; McComas and Mossawy, 1965). In the latter condition there is evidence that the membrane permeability may be raised not only for ions such as sodium and potassium, but also for larger molecules such as aldolase (Zierler, 1961). It is, therefore, tempting to attribute the low values of resting potential in the two cases of dystrophia myotonica to the presence of dystrophic changes in the muscle fibres rather than to assume that the values result from a more specific membrane defect.

In myotonia congenita, on the other hand, the present findings indicate that the resting potential is normal and confirm the observations of Riecker et al. (1964) who used a cannula technique to study 50 fibres in five patients. The study of Hofmann et al. (1966) reveals an apparent discrepancy, since the resting potentials were severely reduced in their youngest patient in whom a diagnosis of myotonia congenita had been made. However, in this patient 
there was histological evidence of dystrophy and, using the argument presented above, it seems possible that this may have accounted for the results. The findings of Norris (1962) are more difficult to interpret, since, although the resting potentials were not significantly reduced in his two patients, one of whom had myotonia congenita, the measurements are low compared with control values reported by other workers for in vivo studies in man (Johns, 1960; Creutzfeldt, Abbott, Fowler, and Pearson, 1963; Riecker et al., 1964; Goodgold and Eberstein 1966; See Table 2 of McComas et al., 1968). The ethical problem involved in obtaining satisfactory control values using the technique of surgical exposure of muscle is clearly a formidable one (McComas et al., 1968) and, in fact two, if not three, of Norris's four control subjects had conditions in which the resting membrane potential might well have been abnormal (dermatomyositis, familial periodic paralysis, and benign congenital hypotonia respectively).

MEMBRANE HYPEREXCITABILITY At the present time the nature of the disorder responsible for the characteristic hyperexcitability of the myotonic muscle fibre membrane remains obscure. Nevertheless, there are at least five possible mechanisms which deserve consideration and each of these will now be discussed in relation to the findings of the present study.

1. Proximity of resting and critical membrane potentials If the resting membrane potential were very close to the threshold or 'critical' level for the initiation of impulses, then there would be an increased likelihood of excitation occurring. Impulses could arise spontaneously or following any induced change in membrane properties sufficient to depolarize the membrane by a few millivolts-for example, after cooling or mechanical damage. There is evidence to suggest that such a mechanism may exist in a proportion of fibres in hereditary mouse dystrophy (McComas and Mossawy, 1966). In the present study we have explored this possibility by measuring the critical membrane depolarization, and our results indicate that this hypothesis cannot account for the hyperexcitability in dystrophia myotonica and is unlikely to be the underlying defect in myotonia congenita. Thus the reduction in resting membrane potential in dystrophia myotonica is matched by a corresponding fall in critical membrane potential with the result that the critical membrane depolarization remains unchanged (see Results).

The position regarding myotonia congenita is less clearly defined. Some of the fibres did have reduced critical membrane depolarizations, in one fibre as low as $\mathbf{~ m V}$; in all these fibres, however, there was a progressive fall in resting potential such as to suggest that the fibres were damaged. On the other hand, there was no doubt that in other myotonic fibres the critical membrane potential was normal (see Fig. 2, lower) and we are inclined to attach more significance to this observation than to the previous one.

2. Reduction in chloride permeability of membrane Bryant (1962) showed that normal mammalian skeletal muscle fibres would fire spontaneously if the chloride conductance was artificially lowered. In a subsequent study, Lipicky and Bryant (1966) produced evidence to show that the chloride permeability was, in fact, reduced in goat myotonia. Although we have not been able to measure the chloride permeability in our study, because of its in vivo nature, there are two other measurements which give indirect evidence of its magnitude; these are the membrane time constant $\left(\mathrm{T}_{\mathrm{m}}\right)$ and the input resistance $\left(R_{i n}\right)$. If the chloride permeability $\left(P_{C l}\right)$ had been reduced then both these parameters should have increased.

Thus $\mathbf{P}_{\mathbf{m}}=\mathbf{P}_{\mathrm{Na}}+\mathbf{P}_{\mathrm{K}}+\mathbf{P}_{\mathbf{C l}}$

and $P_{m} \propto G_{m}$. (cf. Katz, 1966, p. 72)

$$
\begin{aligned}
& \text { Also } \frac{1}{\mathbf{G}_{\mathrm{m}}} \propto \mathbf{R}_{\mathrm{m}}, \\
& \text { and } \mathbf{R}_{\mathrm{m}} \cdot \mathbf{C}_{\mathrm{m}}=\mathrm{T}_{\mathrm{m}} \\
& \text { Similarly } \sqrt{\mathbf{R}_{\mathrm{m}}} \propto \mathbf{R}_{\mathrm{in}}
\end{aligned}
$$

(Where $R_{m}$ is the specific membrane resistance, $C_{m}$ is the membrane capacitance, $G_{m}$ is the membrane conductance, and $P_{m}$ is the net ionic permeability of the membrane.) In fact, however, both the input resistances and the membrane time constants in dystrophia myotonica were reduced compared with the normal values.

In the study of Hofmann et al. (1966) electrotonic potentials were studied in 12 fibres of patients with dystrophia myotonica; the membrane time constants were found to range from 9.2 to $25 \mathrm{msec}$. Although these values were considerably higher than those of the present investigation, they were normal for intercostal muscle (Elmqvist, Hofmann, Kugelberg, and Quastel, 1964).

We have not been able to measure the membrane constants in myotonia congenita and our only indication of membrane permeability in that condition is the finding of normal resting membrane potentials. This observation suggests that the transmembrane concentrations of sodium and potassium are normal and that the relative permeability of the membrane for these two ions is also unchanged; unfortunately, however, it gives no indication of chloride permeability (Hodgkin, 1958).

3. Post-hyperpolarization excitation At the conclusion of an action potential the potassium permeability of the fibre membrane is high and the mem- 
brane potential is therefore equal to the potassium equilibrium potential. If the resting membrane potential is lower than this, because the $\mathrm{Na}: \mathrm{K}$ permeability ratio is abnormally high, the membrane potential will subsequently decline to the resting value; this depolarization may activate the regenerative sodium conductance mechanism responsible for the action potential. Such a mechanism has been advanced to explain the genesis of fibrillation potentials in denervated muscle (Lüllmann, 1960) and the autorhythmicity of cardiac muscle fibres (Dudel and Trautwein, 1958). However, the available evidence suggests that it is unlikely to be involved in myotonia.

First, quiescent fibres in myotonia congenita were found to have normal resting potentials, as previously noted by Riecker et al. (1964). We have also attributed normal resting potentials to active fibres, but it could be argued that the true resting potential is not the post-spike level but a somewhat lower one and the feature which we have termed 'pre-potential' is actually a positive after-potential. Although our own observations indicate that in such fibres the resting potential on entry is normal before the first spike, evidence of post-spike hyperpolarization has been found by Hofmann et al. (1966).

On the other hand it is curious that myotonia is less common in dystrophia myotonica than in myotonia congenita, even though the resting potentials are very significantly reduced in the former condition. It would also be necessary to explain why myotonia is not usually evident in denervated muscle if the underlying membrane defect were the same in both conditions.

4. Membrane calcium deficiency Another hypothesis to account for myotonia depends on the wellknown observation that biological membranes become unstable in the absence of calcium ions (for example, Adrian and Gelfan, 1933; Arvanitaki, 1939). However, the careful studies of Hofmann et al., (1966) have shown that myotonic features of the muscle membrane are only partly suppressed when the extracellular calcium concentration is trebled, so that this explanation also appears unsatisfactory.

5. Structural defect of membrane McComas and Mossawy (1965) have suggested that the myotonic membrane is structurally defective and might even 'rupture'. In this way myotonia could occur whenever the fibre membrane was deformed-for example, by percussion of the muscle belly or insertion of a needle electrode, or during contraction and relaxation. In this study, further evidence of the exquisite mechanical sensitivity of the fibres has been found in the invariable presence of pre-potentials before the myotonic spikes (see Results). The pre-potentials also indicate that the relative permeability of the membrane for sodium and potassium is changing but they provide no clue as to the way in which this change is effected.

In conclusion, we have not been able to determine the cause of the myotonic phenomenon. However, on the negative side we can say, with some confidence, that it is not due to proximity of the resting and critical membrane potentials. Again, we have found no evidence of a reduced chloride permeability in dystrophia myotonica, although the situation for myotonia congenita remains unknown. A calcium effect has been explored and largely discounted by Hofmann et al. (1966) and the evidence for posthyperpolarization activation seems unconvincing. The concept of a structural defect in the membrane has also been considered, but no attempt has been made to specify either the nature or the magnitude of the postulated abnormality.

\section{SUMMARY}

1. An electrophysiological examination of myotonic muscle has been made using the technique of intracellular recording with micro-electrodes. Two patients with myotonia congenita and two with dystrophi myotonia were examined.

2. The resting membrane potential was abnormallo low in dystrophia myotonica and this has bees attributed to the dystrophic features of the condition 0 In contrast to this, the resting membrane potential in myotonia congenita was found to be normal.

3. The results of intracellular stimulation have beet. considered in relation to various hypothetic mechanisms which could cause membrane hyper- $\infty$ excitability in myotonia.

We must first thank the four patients for their kind cooperation in this study, and secondly Dr. J. N. Walton who has given continued encouragement and support. We $\frac{\mathrm{O}}{\mathbb{D}}$ are also indebted to Dr. J. B. Foster for permission to investigate one of his patients. Assistance was received $\overline{\bar{O}}$ from Dr. D. Gardner-Medwin and we also wish to 3 acknowledge generous help received from Dr. W. G. Bradley, Dr. H. B. Ghosh, Mr. C. A. Wallace, Mrs. Y. Chisholm, Mrs. S. Uldall, and the neurosurgical nursing and technical staffs. The project received financial support from the Medical Research Council, the Muscular Dystrophy Group of Great Britain, and the Muscular Dystrophy Associations of America, Inc.

\section{REFERENCES}

Adrian, E. D., and Gelfan, S. (1933). Rhythmic activity in skeletal I muscle fibres. $J$. Physiol. (Lond.), 78, 271-287.

Arvanitaki, A. (1939). Recherches sur la réponse oscillatoire locale de l'axone géant isolé de Sepia. Arch. int. Physiol., 49, 209-256. N

Brown, G. L., and Harvey, A. M. (1939). Congenital myotonia in the goat. Brain, 62, 341-363.

Bryant, S. H. (1962). Muscle membrane of normal and myotonic goats in normal and low external chloride. Fed. Proc., 21, 312. 
Creutzfeldt, O. D., Abbott, B. C., Fowler, W. M., and Pearson, C. M. (1963). Muscle membrane potentials in episodic adynamia. Electroenceph. clin. Neurophysiol., 15, 508-519.

Denny-Brown, D., and Nevin, S. (1941). The phenomenon of myotonia. Brain, 64, 1-18.

Dudel, J., and Trautwein, W. (1958). Der Mechanismus der automatischen rhythmischen Impulsbildung der Herzmuskelfaser. Pflügers Arch. ges. Physiol., 267, 553-565.

Elmqvist, D., Hofmann, W. W., Kugelberg, J., and Quastel, D. M. J. (1964). An electrophysiological investigation of neuromuscular transmission in myasthenia gravis. J. Physiol. (Lond.), 174, 417-434.

Floyd, W. F., Kent, P., and Page, F. (1955). An electromyographic study of myotonia. Electroenceph. clin. Neurophysiol., 7, 621630.

Goodgold, J., and Eberstein, A. (1966). Transmembrane potentials of human muscle cells in vivo. Exp. Neurol., 15, 338-346.

Hodgkin, A. L. (1958). Ionic movements and electrical activity in giant nerve fibres. Proc. Roy. Soc. B., 148, 1-37.

Hofmann, W. W., Alston, W., and Rowe, G. (1966). A study of individual neuromuscular junctions in myotonia. Electroenceph. clin. Neurophysiol., 21, 521-537.

- - and Rowe, G. (1966). Electrophysiological study of myotonia. Nature (Lond.), 212, 954.

Johns, R. J. (1960). Micro-electrode studies of muscle membrane potentials in man. Res. Publ. Ass. nerv. ment. Dis., 38, 704-713.

Katz, B. (1966). Nerve, Muscle and Synapse. McGraw-Hill, New York.

Kleeman, F. J., Partridge, L. D., and Glaser, G. H. (1961). Resting potential and muscle fibre size in hereditary mouse dystrophy. Amer. J. phys. Med., 40, 219-224.

Kolb, L. C. (1938). Congenital myotonia in goats. Bull. Johns Hopk. Hosp., 63, 221-237.

Lenman, J. A. R. (1963). Microelectrode studies in muscle disease, pp. 320-392, in Research in Muscular Dystrophy: Proceedings of the Second Symposium of the Muscular Dystrophy Group. Pitman Medical, London.

Lindsley, D. B., and Curnen, E. C. (1936). An electromyographic study of myotonia. Arch. Neurol. Psychiat. (Chic.), 35, 253-269.
Lipicky, R. J., and Bryant, S. H. (1966). Sodium, potassium and chloride fluxes in intercostal muscle from normal goats and goats with hereditary myotonia. J. gen. Physiol., 50, 89-111.

Ludin, H. F. (1967). Micro-electrode study of normal and dystrophic human muscle, in International Meeting on Electromyography (Abstracts). Glasgow.

Lüllmann, H. (1960). Uber die Ursache spontaner Fibrillationen denervierter Skeletmuskulatur. Klin. Wschr., 38, $1169-1171$.

McComas, A. J., and Mossawy, S. J. (1965). Electrophysiological investigation of normal and dystrophic muscles in mice, pp. 317-341, in Research in Muscular Dystrophy: Proceedings of the Third Symposium of the Muscular Dystrophy Group. Pitman Medical, London.

- - (1966). Excitability of muscle fibre membranes in dystrophic mice. J. Neurol. Neurosurg. Psychiat., 29, 440-445.

—, Mrożek, K., Gardner-Medwin, D., and Stanton, W. H. (1968). Electrical properties of muscle fibre membranes in man. Ibid., 31, 434-440.

McIntyre, A. R., Bennett, A. L., and Brodkey, J. S. (1959). Muscular dystrophy in mice of the Bar Harbor strain. An electromyographic comparison with dystrophia myotonica in man. Arch. Neurol. Psychiat. (Chic.), 81, 678-692.

Norris, F. H. Jr. (1962). Unstable membrane potential in human myotonic muscle. Electroenceph. clin. Neurophysiol., 14, 197 201.

Riecker, G., Dobbelstein, H., Röhl, D., and Bolte, H. D. (1964). Messungen des Membranpotentials einzelner quergestreifter Muskelzellen bei Myotonia congenita (Thomsen). Klin. Wschr., 42, 519-522.

Van der Meulen, J. P., Gilbert G. J., and Kane, C. A. (1961). Familial hyperkalemic paralysis with myotonia. New Engl.J. Med., 264, $1-6$.

Worster-Drought, C., and Sargent, F. (1952). Muscle fasciculation and reactive myotonia in polyneuritis. Brain, 75, 595-600.

Zierler, K. L. (1961). Potassium flux and further observations on aldolase flux in dystrophic mouse muscle. Bull. Johns Hopk. Hosp., 108, 208-215. 